\title{
Calibration of Passive Microwave Polarimeters that Use Hybrid Coupler-Based Correlators
}

\author{
J.R. Piepmeier, Member, IEEE
}

\begin{abstract}
Four calibration algorithms are studied for microwave polarimeters that use hybrid coupler-based correlators: 1) conventional two-look of hot and cold sources, 2) three looks of hot and cold source combinations, 3) two-look with corrclated source, and 4) Your-look combining methods 2 and 3 . The systematic errors are found to depend on the polarimeter component parameters and accuracy of calibration noise temperatures. A case study radiometer in four different remote sensing scenarios was considered in light of these results. Applications for ocean surface salinity, ocean surface winds, and soil moisture were found to be sensitive to different systematic errors. Finally, a standard uncertainty analysis was performed on the four-look calibration algorithm, which was found to be most sensitive to the correlated calibration source.
\end{abstract}

Index Terms-calibration, correlators, microwave radiometry, polarimetry, polarization

\section{INTRODUCTION}

$\mathbf{P}$ ASSIVE microwave polarimetry, or polarimetric radiometry, is relatively new to Earth remote sensing. While it has been quite useful over the last several decades in radio astronomy, only recently has microwave polarimetry been shown to be of utility to sensing geophysical quantities. Ocean surface wind direction remote sensing was indeed enabled by pclarimetric radiometers (e.g., [1], [2]). Additionally, the use of polarimetry for removing the effects of ionospheric Faradiay rotation was proposed in [3] and electronic polarization basis rotation was described in [4]. We define a polarimeter, or polarimetric radiometer, as a radiometer that is designed to measure polarization information beyond the conventional dual-polarized radiometer. For example, a polarimeter might measure the correlation between the verticallyand horizontally-polarized fields, or it might measure $\pm 45^{\circ}$ linear polarization. This information is then used to determine parameters of the polarization ellipse or the Stokes vector. Some examples of Earth science polarimeter architecture can be found in [5] with specific implementation examples in [6] and [2]; for a survey of astronomical polarimetry see [7].

In this paper we are specifically studying the calibration of a hybrid coupler-based polarimeter of the type shown in Fig.1. Dur objective is to determine the effects of calibration algorithms on the systematic errors of the measurement. The polarimeter measures the first three modified Stokes parameters in brightness temperature $T_{v}, T_{h}$, and $T_{U}$. The modified

The aluthor is with the Microwave Instrument Technology Branch at NASA's Goddard Space Flight Center, Grecnbelt, MD 20771 USA. E-mail: jeff.piepmeier@nasa.gov.

This work was supported by the NASA ESE Earth Science Technology Office and NASA's Goddard Space Flight Center.
Stokes vector in brightness temperature is defined:

$$
\left(\begin{array}{l}
T_{v} \\
T_{h} \\
T_{U} \\
T_{V}
\end{array}\right)=\frac{\lambda^{2}}{\eta k B}\left(\begin{array}{c}
\left\langle\left|E_{v}\right|^{2}\right\rangle \\
\left.\left|E_{h}\right|^{2}\right\rangle \\
2 \operatorname{Re}\left\langle E_{v} E_{h}^{*}\right\rangle \\
2 \operatorname{Im}\left\langle E_{v} E_{h}^{*}\right\rangle
\end{array}\right)
$$

where $\lambda$ is the wavelength, $k$ is Boltzmann's constant, $B$ is the detection bandwidth and $\eta$ is the intrinsic impedance. The first two modified Stokes parameters are the vertical and horizontal polarized brightness temperatures measured by conventional dual polarization radiometers. The third Stokes parameter is measured by correlating the vertical and horizontal voltage signals. The hybrid coupler-based polarimeter is based upon the principle of correlation through synthesis of the $\pm 45^{\circ}$ linear polarizations from the vertical and horizontal signals:

$$
\begin{aligned}
& \left\langle\left|E_{p}\right|^{2}\right\rangle=\frac{1}{2}\left\langle\left|E_{v}\right|^{2}\right\rangle+\frac{1}{2}\left\langle\left|E_{h}\right|^{2}\right\rangle+\operatorname{Re}\left\langle E_{v} E_{h}^{*}\right\rangle \\
& \left\langle\left|E_{m n}\right|^{2}\right\rangle=\frac{1}{2}\left\langle\left|E_{v}\right|^{2}\right\rangle+\frac{1}{2}\left\langle\left|E_{h}\right|^{2}\right\rangle-\operatorname{Re}\left\langle E_{v} E_{h}^{*}\right\rangle
\end{aligned}
$$

where the subscripts $p$ and $m$ stand for plus and minus fortyfive degrees, respectively.

We do not include the antenna in this analysis. The antenna polarization effects are assumed to be compensated by the antenna pattem correction process, which is a conventional technique used in radio astronomical polarimetry [7]. This strategy allows design and calibration methodologies each to be separated into two independent parts at the antennaradiometer interface.

The polarimeter can be described by the following forward system equation:

$$
\left(\begin{array}{c}
v_{v} \\
v_{h} \\
v_{p} \\
v_{m}
\end{array}\right)=\left(\begin{array}{ccc}
G_{v v} & 0 & 0 \\
0 & G_{h h} & 0 \\
G_{p v} & G_{p h} & G_{p U} \\
G_{m v} & G_{m h} & G_{m U}
\end{array}\right)\left(\begin{array}{c}
T_{v} \\
T_{h} \\
T_{U}
\end{array}\right)+\left(\begin{array}{c}
o_{v} \\
o_{h} \\
o_{p} \\
o_{m}
\end{array}\right)
$$

where the elements of the left-hand vector are proportional to the average voltage at the detector outputs. We can assume that the cross-polarization terms in rows one and two of the gain matrix are made negligible through the appropriate use of isolators and amplifiers. The radiometer contains two amplifier and filtering chains that form a conventional dual-polarization radiometer used to measured $T_{v}$ and $T_{h}$. In addition, signals from the two chains are combined using a $180^{\circ}$-hybrid coupler and then detected to measure two additional slant-linear polarization brightness temperatures $T_{p}$ (for $+45^{\circ}$ linear or p-pol) and $T_{m}$ (for $-45^{\circ}$ linear or $\mathrm{m}$-pol). The third Stokes parameter can be found from their difference: $T_{U}=T_{p}-T_{m}$. 


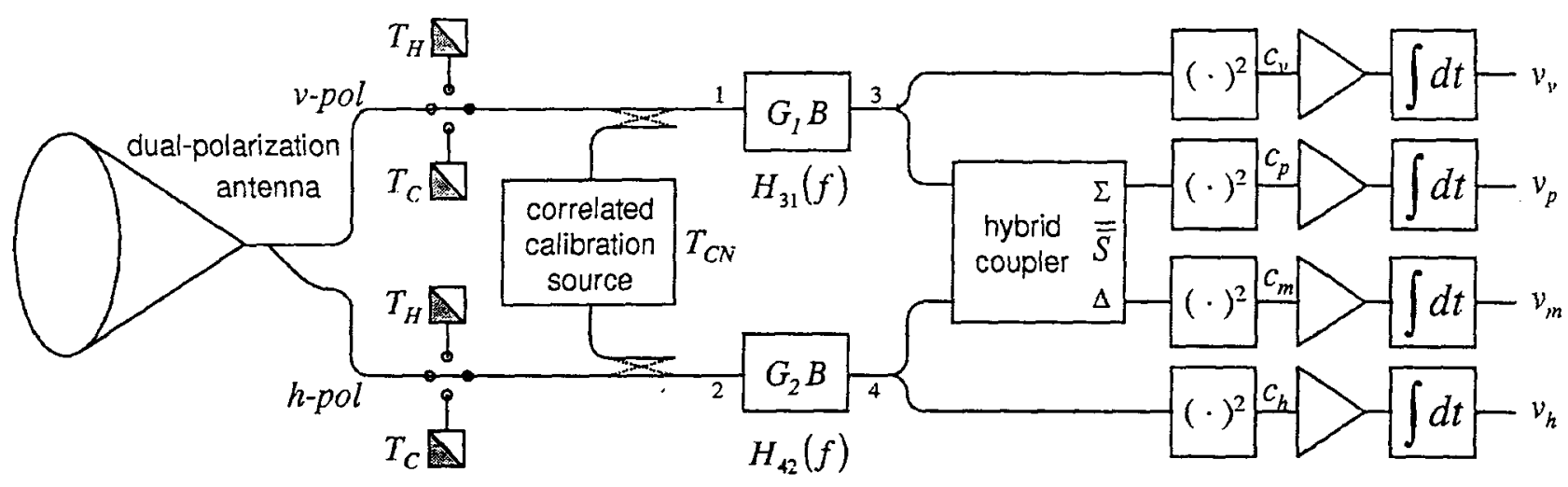

Fig. 1. Block diagram of hybrid coupler-based polarimeter for measuring the first three modified-Stokes parameters $T_{v}, T_{h}$, and $T_{U}$.

To study and quantify the quality of polarimeter calibration, we examine four calibration algorithms using a combination of hot, cold, and correlated calibration sources. Each calibration methodology produces systematic errors that must be controlled through design requirements and affect the polarimeter calibration uncertainty. The following methodology is used to study the four cases. First, a comprehensive forward model of the radiometer is developed in Section $\Pi$. This model is assumed to describe how signals actually propagate through the polarimetric receiver. Second, for each specific calibration case a modified radiometer equation is chosen such that it has a reduced number of degrees-of-freedom commensurate with the chosen calibration scheme. For example, if only a pair of hot and cold calibration looks are provided (two degreeof-freedom), then the system can only be modeled with two parameters per channel. This modified equation can be inverted to arrive at an estimation of $T_{U}$ called $\hat{T}_{U}$. Next, calibration equations are formed for computing the gains and offsets present within the modified radiometer equation. Into these equations, expressions for the radiometer outputs are inserted based on the actual forward model. Thus, the gains and offsets are found in terms of radiometer hardware parameters and calibration noise temperatures. Finally, these gains and offsets are inserted into the $\hat{T}_{U}$ equation and the systematic errors are found. In Section IV three scenarios (ocean surface salinity, ocean surface wind vector, and soil moisture remote sensing) using the polarimeter are considered. Given each of these three scenarios, quantitative examples of systematic errors are given for different calibration algorithms and a quantitative uncertainty analysis is completed using the guidelines in [8].

\section{Polarimeter Forward Model}

In this section, the gain and offset parameters in (3) are computed based on the microwave properties of the radiometer circuits. Specifically, the parameters can be found in terms of the amplitude and phase behavior of the amplifier chains and hybrid coupler. This analytically-based model can then be used to predict the actual behavior of the radiometer and is useful for determining the performance of a chosen calibration method, which is done in the remaining sections.
The amplifier and filter chains have arbitrary transfer functions $H_{31}(f)$ and $H_{42}(f)$. The effects of the signal splitters, which couple the signals to the hybrid coupler, can be included in the transfer functions and do not need to be explicitly noted. The hybrid coupler is assumed to have negligible frequency dependence and to be well matched over the band of interest, which is not unreasonable for operation only over several percent of bandwidth. It can be shown that such a hybrid coupler, if reciprocal and lossless, has the scattering parameter matrix of the form:

$$
\overline{\bar{S}}=\left(\begin{array}{cccc}
0 & 0 & s & \sqrt{1-s^{2}} \\
0 & 0 & \sqrt{1-s^{2}} e^{j \psi} & -s e^{j \psi} \\
s & \sqrt{1-s^{2}} e^{j \psi} & 0 & 0 \\
\sqrt{1-s^{2}} & -s e^{j \psi} & 0 & 0
\end{array}\right)
$$

The reference phase planes are adjusted so that the scattering parameter $s$ is real and positive, and $10 \log _{10} \frac{s^{2}}{1-s^{2}}$ is the amplitude imbalance of the hybrid coupler in decibels. Amplitude imbalance is plotted versus the scattering parameter $s$ (normalized by $2^{-1 / 2}$ ) in Fig.2. Ideally $s=2^{-1 / 2}$ and the imbalance is $0 \mathrm{~dB}$. The phase term $\psi$ is the phase imbalance and ideally is equal to zero.

The mean voltage out of each detector can be found using the above models:

$$
\begin{aligned}
v_{v}= & c_{v} k T_{v} G_{1} B+o_{v} \\
v_{h}= & c_{h} k T_{h} G_{2} B+o_{h} \\
v_{p}= & c_{p} k T_{v} s^{2} G_{1} B+c_{p} k T_{h}\left(1-s^{2}\right) G_{2} B \\
& +c_{p} k T_{U} s \sqrt{1-s^{2}} \int_{-\infty}^{+\infty} H_{31}(f) H_{42}^{*}(f) e^{j \psi \operatorname{sgn}(f)} d f \\
& +o_{p} \\
v_{m}= & c_{m} k T_{v}\left(1-s^{2}\right) G_{1} B+c_{m} k T_{h} s^{2} G_{2} B \\
& -c_{m} k T_{U} s \sqrt{1-s^{2}} \int_{-\infty}^{+\infty} H_{31}(f) H_{42}^{*}(f) e^{j \psi \operatorname{sgn}(f)} d f \\
& +o_{m}
\end{aligned}
$$

where $c_{\beta}$ is the detector sensitivity for polarization channel $\beta$. The signum function in the exponent makes the phase-shift factor Hermitian. The gain-bandwidth products $G_{n} B, n=1$ 


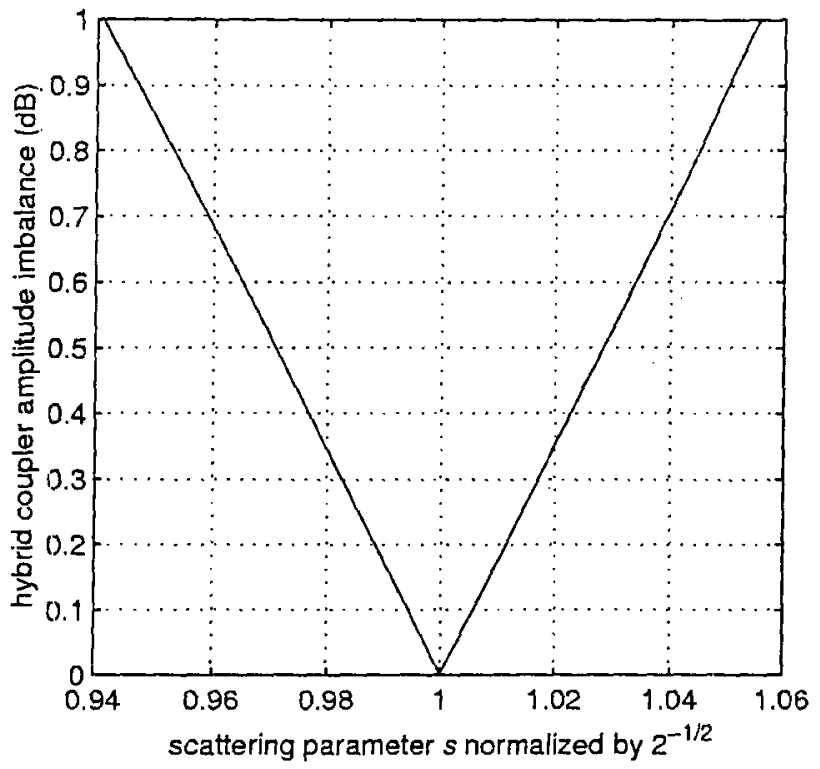

Fig. 2. Hybrid coupler amplitude imbalance in decibels versus the scattering parameter $s$ normalized by $2^{-1 / 2}$.

or 2, are defined as:

$$
\begin{aligned}
& G_{1} B=\int_{-\infty}^{\infty}\left|H_{31}(f)\right|^{2} d f \\
& G_{2} B=\int_{-\infty}^{\infty}\left|H_{42}(f)\right|^{2} d f
\end{aligned}
$$

The gain of channel two can be referenced to channel one using a gain imbalance $g$ :

$$
G_{2}=g G_{1}
$$

where $\left|\log _{10}(g)\right|$ is the gain imbalance in decibels. The offsets resulting from detected receiver noise $T_{n, R X}$ are:

$$
\begin{aligned}
o_{v} & =c_{v} k T_{1, R X} G_{1} B \\
o_{h} & =c_{v} k T_{2, R X} G_{2} B \\
o_{p} & =c_{p} k T_{1, R X} s^{2} G_{1} B+c_{p} k T_{2, R X}\left(1-s^{2}\right) G_{2} B \\
o_{m} & =c_{m} k T_{1, R X}\left(1-s^{2}\right) G_{1} B+c_{m} k T_{2, R X} s^{2} G_{2} B
\end{aligned}
$$

The integral in the expressions for the polarimetric channels $v_{p}$ and $v_{m}$ is essentially an inner product of $H_{31}$ and $H_{42 .}$ and by the Cauchy-Schwarz theorem, we can write:

$$
\alpha_{e} B \sqrt{G_{1} G_{2}}=\int_{-\infty}^{+\infty} H_{31}(f) H_{42}^{*}(f) e^{j \psi \operatorname{sgn}(f)} d f
$$

where $\alpha_{e} \leq 1$ is a bandpass equalization efficiency. It is affected by the differential phase and amplitude variations betweer the amplifier chains and by the phase imbalance of the hybrid coupler. A similar expression arises in radio interferometry and is similarly affected by the channel characteristics [ 9 ]. It is also effected by decorrelation caused by a group-delay difference (so-called fringe washing). Here we assume that path lengths are equalized to cancel this effect.

While $\alpha_{e}$ is determined by the combined effects of phase and amplitude variations, three different modes that affect its value can be identified: 1) hybrid coupler phase imbalance and/or uniform amplifier chain phase imbalance, 2) amplifier chain random phase differences, and 3) amplifier chain passband ripple differences. Below, the parameter $\alpha_{e}$ is computed for each of these modes taken independently. First, hybrid coupler phase imbalance $(\psi)$ and/or constant phase difference between amplifier chains $(\phi)$ would appear as a cosine factor:

$$
\alpha_{e}(\phi, \psi)=\cos (\phi+\psi)
$$

The phase difference between the two amplifier chains across the passband, however, is rarely constant. If the phase difference is modeled as a uniform random variable with mean $\phi$ and limits $\phi \pm \Delta \phi$, then $\alpha_{e}$ can be found from the expected value of the inner-product integral (9):

$$
\left\langle 2 \operatorname{Re} \int_{0}^{+\infty} H_{31}(f) H_{42}^{*}(f) e^{j \phi} e^{j \phi^{\prime}} d f\right\rangle_{\phi^{\prime}}
$$

where the operator $\langle\cdot\rangle_{\phi^{\prime}}$ is the expected value operator of the uniform random variable $\phi^{\prime}$ over the interval $\pm \Delta \phi$. Carrying out the integration and factoring out the coefficients identifiable with $\alpha_{e}$ yields:

$$
\alpha_{e}(\phi ; \Delta \phi)=\cos \phi \operatorname{sinc} \Delta \phi
$$

The third mode to examine is passband ripple differences. The transfer functions can be parameterized to include sinusoidal amplitude ripple such that

$$
\begin{aligned}
& H_{31}(f)=\left(\frac{G_{1}}{1+\left|\gamma_{1}\right|^{2}}\right)^{\frac{1}{2}} \Pi\left(\frac{|f|-f_{0}}{B}\right) \\
& \times\left(1+\gamma_{1} e^{j \pi \operatorname{sgn}(f) \frac{|f|-f_{0}}{B}(N-1)}\right) \\
& H_{42}(f)=\left(\frac{G_{2}}{1+\left|\gamma_{2}\right|^{2}}\right)^{\frac{1}{2}} \Pi\left(\frac{|f|-f_{0}}{B}\right) \\
& \times\left(1+\gamma_{2} e^{j \pi \operatorname{sgn}(f) \frac{|f|-f_{0}}{B}(N-1)}\right)
\end{aligned}
$$

where $\gamma_{n}$ is the ripple amplitude and $N$ is the number of poles of a representative odd-order Chebyshev filter. The peakto-peak ripple in decibels is $20 \log _{10} \frac{1+\left|\gamma_{n}\right|}{1-\left|\gamma_{n}\right|}$. (Note that these transfer functions still satisfy (6).) By substituting the above into the inner-product integral, we find that

$$
\alpha_{e}\left(\gamma_{1}, \gamma_{2}\right)=\frac{1+\operatorname{Re} \gamma_{1} \gamma_{2}^{*}}{\sqrt{\left(1+\left|\gamma_{1}\right|^{2}\right)\left(1+\left|\gamma_{2}\right|^{2}\right)}}
$$

and is bounded

$$
\begin{aligned}
& \frac{1}{\sqrt{\left(1+\left|\gamma_{1}\right|^{2}\right)\left(1+\left|\gamma_{2}\right|^{2}\right)}} \\
& \leq \alpha_{e}\left(\gamma_{1}, \gamma_{2}\right) \leq \\
& \frac{1+\left|\gamma_{1}\right|\left|\gamma_{2}\right|}{\sqrt{\left(1+\left|\gamma_{1}\right|^{2}\right)\left(1+\left|\gamma_{2}\right|^{2}\right)}}
\end{aligned}
$$

or if the ripple of the two channels is of equal amplitude $|\gamma|$, then

$$
\frac{1}{1+|\gamma|^{2}} \leq \alpha_{e}(\gamma) \leq 1
$$






Fig. 3. Dependence of $\alpha_{e}(\gamma)$ on peak-to-peak amplitude ripple of the passband response of the radiometer channels. A 2.5-dB ripple results in up to $2 \%$ reduction in efficiency.

Fig. 3 shows the dependence of $\alpha_{e}$ on peak-to-peak amplinude ripple in $\mathrm{dB}$.

Finally, the polarimeter forward model is found by rewriting (5) as a matrix equation of the form in (3) and is shown at the bottom of the page (17). Throughout the remainder of this paper this forward model is used to describe how the polarimeter actually operates. It captures nonidealities such as differences in gain and phase of the amplifier and filter chains, and phase and amplitude imbalance of the hybrid coupler. Using this model, we determine the systematic errors of the polarimeter under four different calibration algorithms or cases in the following section.

\section{Calibration}

Total power radiometers are conventionally calibrated using two sources of differing brightness or noise temperatures [10]. The polarimeter considered here is essentially four totalpower radiometers, each sensitive to a different polarization, and is tempting to be calibrated as such. The four radiometers, however, are not entirely decoupled, and the calibration scheme can should take this into account. Here, we consider four calibration algorithms and examine their effects on the systematic errors of the polarimeter. The errors are manifest as either or both of polarimetric inefficiency or depolarization (see [7]) and cross-polarization coupling depending upon the choice of calibration method. Case 1 is the conventional twolook method. Case 2 is a three-look method that includes varying combinations of hot and cold sources. Case 3 is also a three look method but combines Case 1 with a correlated calibration source. Finally, Case 4 uses the correlated source in conjunction with the three looks of Case 2 for a complete characterization of the polarimeter. In all four cases, we assume that the two total-power radiometers measuring $T_{v}$ and $T_{h}$ can best be calibrated using the conventional two-look technique. We, therefore, only consider the calibration of $T_{U}$.

\section{A. Case 1: Conventional hot and cold calibration}

The first case is simply the conventional two-look calibration method used with standard total-power radiometers. Each channel is calibrated independently using a hot look and cold look by vicwing two calibrators with differing brightness or noise temperatures. Because only two calibration states are used, only two calibration parameters can be found for each channel. The following is used for the Case- 1 radiometer model for measuring $T_{p}$ and $T_{m}$ :

$$
\left(\begin{array}{c}
v_{p} \\
v_{m}
\end{array}\right)=\frac{1}{2}\left(\begin{array}{ccc}
G_{p} & G_{p} & G_{p} \\
G_{m} & G_{m} & -G_{m}
\end{array}\right)\left(\begin{array}{c}
T_{v} \\
T_{h} \\
T_{U}
\end{array}\right)+\left(\begin{array}{c}
o_{p} \\
o_{m}
\end{array}\right)
$$

As is seen above, only two parameters are needed for each channel: $G_{p}$ and $o_{p}$ for the $+45^{\circ}$ polarization (p-pol) and $G_{m}$ and $o_{m}$ for the $-45^{\circ}$ polarization (m-pol). Solving for $T_{U}$ yields the estimate $\hat{T}_{U}$ :

$$
\hat{T}_{U}=\frac{v_{p}-o_{p}}{G_{p}}-\frac{v_{m}-o_{m}}{G_{m}}
$$

The two calibrators are implemented as a matched load for the cold noise temperature $T_{C}$ and an additional coupled noise diode for the hot noise temperature $T_{H}$ as seen in Fig. 1. For the p-pol channel, the calibration equation is

$$
\left(\begin{array}{l}
v_{p, C} \\
v_{p, H}
\end{array}\right)=\left(\begin{array}{ll}
T_{C} & 1 \\
T_{H} & 1
\end{array}\right)\left(\begin{array}{l}
G_{p} \\
o_{p}
\end{array}\right)
$$

or solving for $G_{p}$ and $o_{p}$

$$
\begin{aligned}
G_{p} & =\frac{v_{p, H}-v_{p, C}}{T_{H}-T_{C}} \\
o_{p} & =\frac{T_{H} v_{p, C}-T_{C} v_{p, H}}{T_{H}-T_{C}}
\end{aligned}
$$

The noise temperatures $T_{C}$ and $T_{H}$ are the nominal noise temperatures (including errors) of the calibration sources.

Now, if the actual observed voltages as calculated by the complete forward model (17) are substituted into the gain and

$$
\left(\begin{array}{c}
v_{v} \\
v_{h} \\
v_{p} \\
v_{m}
\end{array}\right)=\left(\begin{array}{ccc}
c_{v} G_{1} k B & 0 & 0 \\
0 & c_{h} G_{2} k B & 0 \\
c_{p} s^{2} G_{1} k B & c_{p}\left(1-s^{2}\right) G_{2} k B & c_{p} s \sqrt{1-s^{2}} \alpha_{e} k B \sqrt{G_{1} G_{2}} \\
c_{m}\left(1-s^{2}\right) G_{1} k B & c_{m} s^{2} G_{2} k B & -c_{m} s \sqrt{1-s^{2}} \alpha_{e} k B \sqrt{G_{1} G_{2}}
\end{array}\right)\left(\begin{array}{c}
T_{v} \\
T_{h} \\
T_{U}
\end{array}\right)+\left(\begin{array}{c}
o_{v} \\
o_{h} \\
o_{p} \\
o_{m}
\end{array}\right)
$$


offset equations, the estimate $\hat{T}_{U}$ can be found in terms of the input Stokes vector. First, we find that

$$
\begin{aligned}
& v_{p, C}=c_{p} k B\left(s^{2} G_{1}+\left(1-s^{2}\right) G_{2}\right) T_{C}^{\prime} \\
& v_{p, H}=c_{p} k B\left(s^{2} G_{1}+\left(1-s^{2}\right) G_{2}\right) T_{H}^{\prime}
\end{aligned}
$$

where the primed temperatures indicate the true noise temperatures of the calibration sources). Substituting this into the gain and offset expressions:

$$
\begin{aligned}
G_{p}= & c_{p} k B\left(s^{2} G_{1}+\left(1-s^{2}\right) G_{2}\right) \frac{T_{H}^{\prime}-T_{C}^{\prime}}{T_{H}-T_{C}} \\
o_{p}= & c_{p} k B\left(s^{2} G_{1} T_{1, R X}+G_{2}\left(1-s^{2}\right) T_{2, R X}\right. \\
& \left.\quad+\left(s^{2} G_{1}+\left(1-s^{2}\right) G_{2}\right) \frac{\left(T_{H} T_{C}^{\prime}-T_{C} T_{H}^{\prime}\right)}{T_{H}-T_{C}}\right)
\end{aligned}
$$

and similarly for the $\mathrm{m}$-pol channel:

$$
\begin{array}{rl}
G_{m}=c_{m} k B\left(\left(1-s^{2}\right) G_{1}+s^{2} G_{2}\right) & \frac{T_{H}^{\prime}-T_{C}^{\prime}}{T_{H}-T_{C}} \\
D_{m}=c_{m} & k B\left(\left(1-s^{2}\right) G_{1} T_{1, R X}+s^{2} G_{2} T_{2, R X}\right. \\
& \left.+\left(\left(1-s^{2}\right) G_{1}+s^{2} G_{2}\right) \frac{\left(T_{H} T_{C}^{\prime}-T_{C} T_{H}^{\prime}\right)}{T_{H}-T_{C}}\right)
\end{array}
$$

Going the next step, substitute these into $\hat{T}_{U}$ :

$$
\begin{gathered}
\hat{T}_{U}=\frac{\sqrt{g}(1+g) s \sqrt{1-s^{2}}}{\left(s^{2}+\left(1-s^{2}\right) g\right)\left(\left(1-s^{2}\right)+s^{2} g\right)} \frac{T_{H}-T_{C}}{T_{H}^{\prime}-T_{C}^{\prime}} \alpha_{e} T_{U} \\
+\frac{g\left(2 s^{2}-1\right)}{\left(s^{2}+\left(1-s^{2}\right) g\right)\left(\left(1-s^{2}\right)+s^{2} g\right)} \frac{T_{H}-T_{C}}{T_{H}^{\prime}-T_{C}^{\prime}}\left(T_{v}-T_{h}\right)
\end{gathered}
$$

Here the inadequacies of conventional two-look calibration are seen quite clearly. First, there is a gain term affecting $T_{U}$, part of which is directly proportional to the bandpass equalization efficiency $\alpha_{e}$. As will be seen in Case 3, this error can be removed by using a correlated calibration source. Amplitude imbalance in both the receiver channels and the hybrid coupler also affect the gain of $T_{U}$ or the polarimetric efficiency, as it is called in the astronomical polarimetry literature.

The other error is cross polarization contamination. Amplitude imbalance in the hybrid coupler actually causes mixing of the second (not modified) Stokes parameter $\left(T_{Q}=T_{v}-T_{h}\right)$ into $\hat{T}_{U}$. This is easily shown by taking $(25)$ and setting all parameters to their ideal values (e.g., $g=1$ ) except for $s$, which yields:

$$
\hat{\Gamma}_{U}(s)=\left(2 s^{2}-1\right)\left(T_{v}-T_{h}\right)+2 s \sqrt{1-s^{2}} T_{U}
$$

Fig. 4 shows the amount of $T_{Q}$ mixing into $\hat{T}_{U}$ as a function of hybrid coupler amplitude imbalance. There is about $11.5 \%$ mixing per decibel of imbalance. The next calibration method Case 2 uses independent hot and cold looks to remove crosspolarization mixing.

\section{B. Case 2: Independently applied hot and cold looks}

In situations where the cross-polarization mixing effect dominates the overall uncertainty (as in (26)), a third calibration state can be added to Case 1 . In addition to the hot

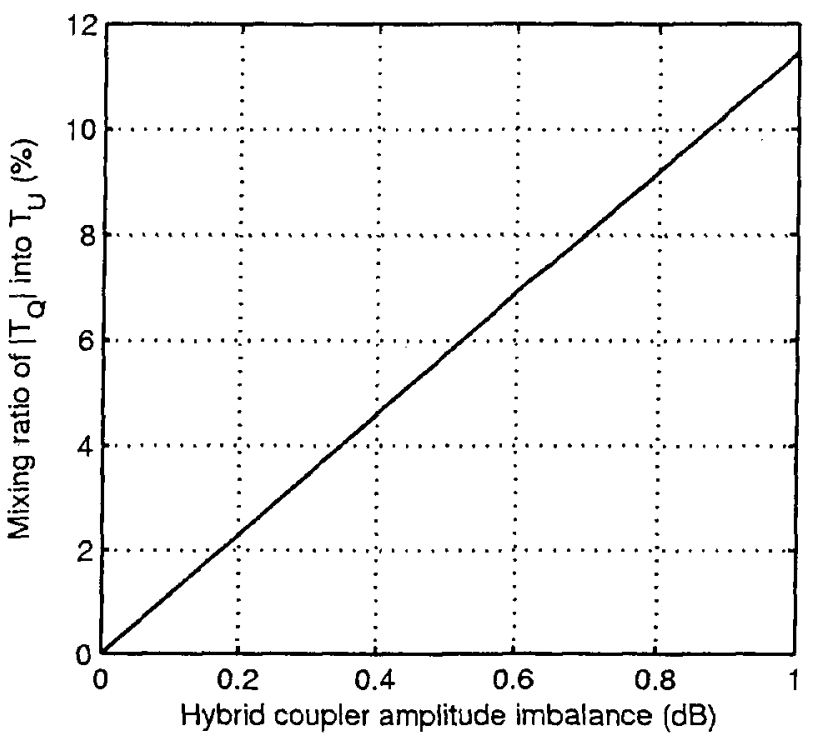

Fig. 4. Mixing ratio of $T_{Q}$ into $T_{U}$ due to hybrid coupler imbalance for Case 1 (conventional two-look calibration).

and cold looks, the third state is one channel viewing hot with the other viewing cold. This third degree of freedom allows the radiometer model to be slightly more complex than (18). The case- 2 radiometer model is:

$$
\left(\begin{array}{c}
v_{p} \\
v_{m}
\end{array}\right)=\frac{1}{2}\left(\begin{array}{ccc}
G_{p v} & G_{p h} & \sqrt{G_{p v} G_{p h}} \\
G_{m v} & G_{m h} & -\sqrt{G_{p v} G_{p h}}
\end{array}\right)\left(\begin{array}{c}
T_{v} \\
T_{h} \\
T_{U}
\end{array}\right)+\left(\begin{array}{c}
o_{p} \\
o_{m}
\end{array}\right)
$$

The differentiation of the gain terms explicitly includes the contributions of the vertical and horizontal polarizations to the p-pol and $\mathrm{m}$-pol channels. Solving for the radiometer equation to find the estimate $\hat{T}_{U}$ :

$$
\begin{gathered}
\hat{T}_{U}=\frac{\sqrt{G_{p h} G_{p v}}}{G_{m h} G_{m v}+G_{p h} G_{p v}}\left(v_{p}-o_{p}-\hat{T}_{h} G_{p h}-\hat{T}_{v} G_{p v}\right) \\
-\frac{\sqrt{G_{m h} G_{m v}}}{G_{m h} G_{m v}+G_{p h} G_{p v}}\left(v_{m}-o_{m}-\hat{T}_{h} G_{m h}-\hat{T}_{v} G_{m v}\right)
\end{gathered}
$$

where the estimates $\hat{T}_{v}$ and $\hat{T}_{h}$ are taken from their respective total power channels. To take advantage of this type of calibration method, the calibration topology of the radiometer must allow for the independent switching of the hot and cold sources. This is readily done by literally implementing the configuration shown in Fig.1. One such way is to use a switch, a matched load, and a coupled noise diode. On the other hand, if the calibration was thru the feedhom, then some sort of externally polarized source would be needed and the explicit separation of the antenna from the receiver would be violated, further complicating the system.

The gains and offsets are found as in Case 1:

$$
\left(\begin{array}{c}
v_{p, C} \\
v_{p, H} \\
v_{p, C H}
\end{array}\right)=\left(\begin{array}{lll}
T_{C} & T_{C} & 1 \\
T_{H} & T_{H} & 1 \\
T_{C} & T_{H} & 1
\end{array}\right)\left(\begin{array}{c}
G_{p v} \\
G_{p m} \\
o_{p}
\end{array}\right)
$$


which when solved yields:

$$
\begin{aligned}
G_{p v} & =\frac{v_{p, H}-v_{p, C H}}{T_{H}-T_{C}} \\
G_{p h} & =\frac{v_{p, C H}-v_{p, C}}{T_{H}-T_{C}}
\end{aligned}
$$

and $o_{p}$ is the same as for Case 1. The m-pol coefficients are can be found in a similar fashion.

Following the same steps performed in the previous section results in the following expression for $\hat{T}_{U}$ :

$$
\begin{aligned}
\hat{T}_{U}= & \alpha_{e} \frac{T_{H}-T_{C}}{T_{H}^{\prime}-T_{C}^{\prime}} T_{U} \\
+ & \sqrt{\frac{1}{g}}\left(\frac{c_{p}^{2}}{c_{m}^{2}+c_{p}^{2}} \sqrt{\frac{s^{2}}{1-s^{2}}}-\frac{c_{m}^{2}}{c_{m}^{2}+c_{p}^{2}} \sqrt{\frac{1-s^{2}}{s^{2}}}\right) \\
& \times\left(T_{v} \frac{T_{H}-T_{C}}{T_{H}^{\prime}-T_{C}^{\prime}}-\hat{T}_{v}-\frac{T_{H} T_{C}^{\prime}-T_{C} T_{H}^{\prime}}{T_{H}^{\prime}-T_{C}^{\prime}}\right) \\
+ & \sqrt{g}\left(\frac{c_{p}^{2}}{c_{m}^{2}+c_{p}^{2}} \sqrt{\frac{1-s^{2}}{s^{2}}}-\frac{c_{m}^{2}}{c_{m}^{2}+c_{p}^{2}} \sqrt{\frac{s^{2}}{1-s^{2}}}\right) \\
& \times\left(T_{h} \frac{T_{H}-T_{C}}{T_{H}^{\prime}-T_{C}^{\prime}}-\hat{T}_{h}-\frac{T_{H} T_{C}^{\prime}-T_{C} T_{H}^{\prime}}{T_{H}^{\prime}-T_{C}^{\prime}}\right)
\end{aligned}
$$

The third calibration state primarily compensates for cross polarization mixing. In essence, any residual offset is due to errors in estimating $T_{v}$ and $T_{h}$. For example, if we simplify (32) by assuming that the amplifier chains are gain balanced $(g=1)$, the detectors have the same sensitivity $\left(c_{m}=c_{p}\right)$, and that the errors in estimated noise temperatures of the calibration looks are negligible, then

$$
\hat{T}_{U}=\alpha_{e} T_{U}+\frac{2 s^{2}-1}{s \sqrt{1-s^{2}}}\left(T_{v}-\hat{T}_{v}-T_{h}+\hat{T}_{h}\right)
$$

where $T_{v}$ and $T_{h}$ are the true brightness temperatures and $\hat{T}_{v}$ and $\hat{T}_{h}$ are the quantities estimated using the radiometer. Note that the residual offset is of similar form to the crosspolarization coupling in Case 1; however, the magnitude is not proportional to $T_{Q}$, but rather the error in the estimate of $T_{Q}$.

As in Case 1, the bandpass equalization efficiency and errors in the hot and cold noise temperatures act on the polarimetric efficiency. In fact, their effect is identical to Case 1. Unlike Case 1, however, the third calibration state has removed the effect of gain imbalance $g$ and coupler amplitude imbalance $s$ on the polarimetric efficiency. The remaining effects only deal in phase. So for a given set of hardware, the third calibration state slightly improves the polarimetric efficiency in addition to nearly complete compensation of cross polarization mixing.

\section{Case 3: Calibration with correlated or polarized source}

On the other hand, in situations where polarimetric efficiency is the dominating consideration, as opposed to cross polarization coupling, the third calibration state can be replaced a with correlated source. Thus, the gain of the third Stokes parameter channel can be explicitly included in the case- 3 radiometer model:

$$
\left(\begin{array}{c}
v_{p} \\
v_{m}
\end{array}\right)=\frac{1}{2}\left(\begin{array}{lll}
\frac{1}{2} G_{p} & \frac{1}{2} G_{p} & G_{p U} \\
\frac{1}{2} G_{m} & \frac{1}{2} G_{m} & G_{m U}
\end{array}\right)\left(\begin{array}{c}
T_{v} \\
T_{h} \\
T_{U}
\end{array}\right)+\left(\begin{array}{c}
o_{p} \\
o_{m}
\end{array}\right)
$$

Inverting this model results in the following estimate of $T_{U}$ :

$$
\hat{T}_{U}=\frac{G_{m}\left(v_{p}-o_{p}\right)-G_{p}\left(v_{m}-o_{m}\right)}{G_{m} G_{p U}-G_{p} G_{m U}}
$$

The gains and offsets are found as before, using p-pol as an example:

$$
\left(\begin{array}{c}
v_{p, C} \\
v_{p, H} \\
v_{p, C N}
\end{array}\right)=\left(\begin{array}{ccc}
T_{C} & 0 & 1 \\
T_{H} & 0 & 1 \\
T_{C}+\frac{1}{2} T_{C N} & \pm T_{C N} & 1
\end{array}\right)\left(\begin{array}{c}
G_{p} \\
G_{p U} \\
o_{p}
\end{array}\right)
$$

The value of the correlated noise temperature $T_{C N}$ depends upon implementation. For example, if the output of a noise diode is split and then coupled into the two channels, $T_{C N}$ is the diode noise temperature reduced by the coupling factor of the directional coupler. The sign of $T_{C N}$ is determined by the relative phase of the coupled signals. When the noise diode is turned on, it adds power to the total power channel and generates a correlated signal mimicking a third Stokes parameter input. Doing so allows $G_{p U}$ to be found:

$$
G_{p U}=\frac{v_{p, C N}-v_{p, C}}{T_{C N}}-\frac{1}{2} G_{p}
$$

where $G_{p}$ is given by 21 . In addition to that for $G_{p}$, the result for $o_{p}$ is identical to Case 1 . The $\mathrm{m}$-pol results are similar.

After substituting the calibration coefficients into the radiometer equation, the following is found for $\hat{T}_{U}$ :

$$
\hat{T}_{U}=\frac{T_{C N}}{T_{C N}^{\prime}} T_{U}+\frac{\sqrt{g}}{(1+g)} \frac{2 s^{2}-1}{s \sqrt{1-s^{2}}} \frac{T_{C N}}{T_{C N}^{\prime}} \alpha_{e}^{-1}\left(T_{v}-T_{h}\right)
$$

Now the calibration of $T_{U}$ is directly dependent upon the accuracy with which the correlated noise temperature is known. The polarimetric efficiency is compensated by the correlated noise source and any error in $T_{C N}$ is transferred directly to $\hat{T}_{U}$. Additionally, like Case $1, T_{Q}$ is mixed directly into $\hat{T}_{U}$ by coupler imbalance. The mixing ratio is within 0.1 percentage points of the case- 1 result shown in Fig. 4.

\section{Case 4: Full calibration}

In the final case, the calibration schemes of the previous cases are combined to fully calibrate the radiometer. In otherwords, four calibration states are used: conventional hot and cold (from Case 1), mixed hot and cold (from Case 2), and correlated noise (from Case 3). With four calibrators come four degrees-of-freedom, allowing the polarimeter to be described by the following forward system equation:

$$
\left(\begin{array}{c}
v_{p} \\
v_{m}
\end{array}\right)=\left(\begin{array}{lll}
G_{p v} & G_{p h} & G_{p U} \\
G_{m v} & G_{m h} & G_{m U}
\end{array}\right)\left(\begin{array}{c}
T_{v} \\
T_{h} \\
T_{U}
\end{array}\right)+\left(\begin{array}{c}
o_{p} \\
o_{m}
\end{array}\right)
$$

Note, the above is exactly the bottom two rows of the complete model in (3). The third Stokes parameter estimate using this system is:

$$
\begin{aligned}
\hat{T}_{U}= & \frac{G_{m U}}{G_{m U}^{2}+G_{p U}^{2}}\left(v_{m}-o_{m}-\hat{T}_{v} G_{m v}-\hat{T}_{h} G_{m h}\right) \\
& +\frac{G_{p U}}{G_{m U}^{2}+G_{p U}^{2}}\left(v_{p}-o_{p}-\hat{T}_{v} G_{p v}-\hat{T}_{h} G_{p h}\right)
\end{aligned}
$$


Three gains and one offset can be found for each channel. Agcin using the p-pol as an example, the calibration equation is:

$$
\begin{gathered}
\left(\begin{array}{c}
v_{p, C} \\
v_{p, H} \\
v_{p, C H} \\
v_{p, C N}
\end{array}\right)= \\
\left(\begin{array}{cccc}
T_{C} & T_{C} & 0 & 1 \\
T_{H} & T_{H} & 0 & 1 \\
T_{C} & T_{H} & 0 & 1 \\
\Gamma_{C}+\frac{1}{2} T_{C N} & T_{C}+\frac{1}{2} T_{C N} & \pm T_{C N} & 1
\end{array}\right)\left(\begin{array}{c}
G_{p v} \\
G_{p m} \\
G_{p U} \\
o_{p}
\end{array}\right)
\end{gathered}
$$

Not surprisingly, the expressions for the first two gain coefficients $G_{p v}$ and $G_{7 n v}$ are the same as for Case 2, $G_{p U}$ is the same as Case 3, and the offset is the same as Case 1. Similar results occur for the m-pol channel. This relation is indicative of the effects of the different calibration sources on the overall instrument calibration. The hot and cold looks help identify the offset, adding the hot-cold combination look differentiates the crosi-polarization gain coefficients; and finally the correlated source, the third Stokes parameter gain coefficient.

The resulting estimate for $T_{U}$ is quite lengthy. It is, however, conceptually quite simple: the accuracy of the estimate is affected by how well the calibration noise temperatures are known and how well the radiometer channels are balanced in gain and phase. The full expression is:

$$
\begin{aligned}
\hat{i}_{U}= & \frac{A c_{p}^{2}+B c_{m}^{2}}{A^{2} c_{p}^{2}+B^{2} c_{m}^{2}} C \frac{T_{C N}}{T_{C N}^{\prime}} T_{U} \\
& +\frac{A}{A^{2}+\frac{c_{m}^{2}}{c_{p}^{2}} B^{2}} \frac{T_{C N}}{T_{C N}^{\prime}} \\
& \times\left(s^{2} D+\left(1-s^{2}\right) g E-\left(s^{2}-s^{2} g+g\right) F\right) \\
& -\frac{B}{\frac{c_{p}^{2}}{c_{m}^{2}} A^{2}+B^{2}} \frac{T_{C N}}{T_{C N}^{\prime}} \\
& \times\left(\left(1-s^{2}\right) D+s^{2} g E-\left(1-s^{2}+s^{2} g\right) F\right)
\end{aligned}
$$

where

$$
\begin{aligned}
& A=C+\frac{1}{2}\left(s^{2}-s^{2} g+g\right)\left(1-\frac{T_{H}^{\prime}-T_{C}^{\prime}}{T_{H}-T_{C}} \frac{T_{C N}}{T_{C N}^{\prime}}\right) \\
& B=C-\frac{1}{2}\left(1-s^{2}+s^{2} g\right)\left(1-\frac{T_{H}^{\prime}-T_{C}^{\prime}}{T_{H}-T_{C}} \frac{T_{C N}}{T_{C N}^{\prime}}\right) \\
& C=s \sqrt{1-s^{2}} \alpha_{e} \sqrt{g} \\
& D=T_{v}-\hat{T}_{v} \frac{T_{H}^{\prime}-T_{C}^{\prime}}{T_{H}-T_{C}} \\
& E=T_{h}-\hat{T}_{h} \frac{T_{H}^{\prime}-T_{C}^{\prime}}{T_{H}-T_{C}} \\
& F=\frac{T_{H} T_{C}^{\prime}-T_{C} T_{H}^{\prime}}{T_{H}-T_{C}}
\end{aligned}
$$

As in the previous cases, we can identify polarimetric efficiency and cross-polarization coupling errors in the expression for $\hat{T}_{i j}$. In the error terms there is an interaction between the radiometer parameters. On one hand, systematic errors in the measurement are caused by errors in calibration noise temperature values, but the effect of these errors can be minimized by careful amplitude and phase balancing of the radiometer. If we assume, however, that all noise and brightness temperatures are known without error, then (42) degenerates to the error free expression $\hat{T}_{U}=T_{U}$. Thus, there is a trade-off between the accuracy of the calibration noise temperature values and the degree to which the radiometer components are balance. Of course it is not possible to know the calibration and brightness temperatures with complete accuracy, so one must consider their effect.

Consider the simplification where the radiometer is perfectly balanced but the calibration noise temperatures and $T_{v}$ and $T_{h}$ estimates are only known with error:

$$
\hat{T}_{U}=\frac{T_{C N}}{T_{C N}^{\prime}} \frac{1}{1+H^{2}} T_{U}+\frac{T_{C N}}{T_{C N}^{\prime}} \frac{H}{1+H^{2}}(D+E-2 F)
$$

where

$$
\begin{aligned}
& D=\left(T_{v}-\hat{T}_{v} \frac{T_{H}^{\prime}-T_{C}^{\prime}}{T_{H}-T_{C}}\right) \\
& E=\left(T_{h}-\hat{T}_{h} \frac{T_{H}^{\prime}-T_{C}^{\prime}}{T_{H}-T_{C}}\right) \\
& F=\frac{T_{H} T_{C}^{\prime}-T_{C} T_{H}^{\prime}}{T_{H}-T_{C}} \\
& H=1-\frac{T_{H}^{\prime}-T_{C}^{\prime}}{T_{H}-T_{C}} \frac{T_{C N}}{T_{C N}^{\prime}}
\end{aligned}
$$

There are basically two main error modes in the above. First, any error in the nominal correlated noise temperature $T_{C N}$ is transferred directly to $\hat{T}_{U}$. This dependence is not unexpected because the correlated noise source was added to compensate for the polarimetric efficiency. Second, errors in the hot and cold noise temperatures and/or $\hat{T}_{v}$ and $\hat{T}_{h}$ cause offsets by altering the contribution of $T_{v}$ and $T_{h}$ to the estimate. At this point, it is more instructive to examine the errors quantitatively by considering a case study radiometer.

\section{APPLICATIONS}

In the previous section we have shown how the combination of imperfect hardware components and selection of calibration method causes systematic errors. In this section the results are applied to several remote sensing problems quantitatively demonstrating the effect of choice of calibration algorithm. Four scenarios are considered: ocean surface salinity (OSS), ocean surface winds (OSW), and soil moisture (SM) with and without antenna polarization basis rotation. In both OSS and SM remote sensing the third Stokes is used for Faraday rotation correction [3], [11]. In OSW, the third Stokes parameter is used in wind direction retrieval, e.g. [1]. For the SM scenario, we add an additional case $S M(b)$ that considers a $45^{\circ}$ polarization basis rotation within the antenna system. Such is relevant to a scanning radiometer antenna that has a fixed feed and a rotating reflector where the polarization basis is continuously rotated and then electronically corrected in post processing [4].

We concentrate on examining the systematic errors and calibration uncertainty arising from systematic effects. We define this component of uncertainty based upon practices of the U.S. National Institute of Standards and Technology (NIST) [12] 
TABLE I

PROPERTIES OF HYPOTHETICAL CASE STUDY RADIOMETER

\begin{tabular}{|c|c|}
\hline \multicolumn{2}{|c|}{ Component parameters } \\
\hline channel gain imbalance & $2 \mathrm{~dB}$ \\
\hline channel phase imbalance & $10^{\circ}$ \\
\hline channel phase variation & $20^{\circ}$ \\
\hline channel amplitude ripple & $2 \mathrm{~dB}$ \\
\hline coupler amplitude imbalance & $0.2 \mathrm{~dB}$ \\
\hline coupler phase imbalance & $5^{\circ}$ \\
\hline \multicolumn{2}{|c|}{ Resulling model parameters } \\
\hline$s$ & 0.700 (from Fig.2) \\
\hline & 1.585 \\
\hline$\alpha_{e}(\gamma)$ & 0.987 (from Fig.3) \\
\hline$\alpha_{e}\left(\psi+\phi_{i} \delta \phi\right)$ & 0.946 Irom (12) \\
\hline$\alpha_{e} \approx \alpha_{e}(\gamma) \alpha_{e}(\psi+\phi ; \delta \phi)$ & 0.934 \\
\hline \multicolumn{2}{|c|}{ Calibration parameters } \\
\hline$\widehat{T_{C}}$ & $250 \mathrm{~K}$ \\
\hline$T_{H}$ & $350 \mathrm{~K}$ \\
\hline$T_{C N}$ & $50 \mathrm{~K}$ \\
\hline
\end{tabular}

and International Standards Organization (ISO) [8]. A systematic effect is something that can produce a systematic error (or bias) in the measurement process. For example, uncertainty in an estimated calibration target emissivity would contribute to the uncertainty arising from systematic effects for a standard microwave radiometer. On the other hand, the random noise in a radiometer measurement due to finite integration time (i.e., NE $\Delta T$ ) does not fall under this category of uncertainty. Another way to consider this uncertainty is that which cannot be reduced by increasing the number of measurements. In our situation, the systematic effects and errors and their associated calibration uncertainty are driven by gain and phase balance behavior of the amplifier chains and the hybrid coupler and choice of calibration method. Performing multiple measurements or increasing integration time is not going to reduce the uncertainty caused by these effects.

The properties of a hypothetical case-study radiometer of the form in Fig. 1 are listed in Table I. These values where chosen to represent some typical performance specifications of commercial off-the-shelf (COTS) components. Of course these values could differ depending upon the radiometer center frequency, bandwidth, and amount of effort invested in design and construction. Nonetheless, they are useful for demonstration purposes.

For the first application, the estimated $T_{U}$ is computed for each of the four different measurement scenarios using the first three calibration algorithms. We assume that the calibration noise temperatures and $T_{v}$ and $T_{h}$ are perfectly known. This allows us to demonstrate numerically the effect of hardware nonidealities and different calibration methods on the measurement result. (Calibration Case 4 was not included because there are no systematic calibration errors under our assumptions.) The results are presented in Table II. A general description of the scene, the incidence angle, observation band, and brightness temperatures are listed in the top rows of the table. The brightness temperatures were determined using published data [10, Figs. 18.2, 18.19, 18.20 and 19.12]. For each calibration case and remote sensing scenario the values
TABLE II

THIRD STOKES PaRAMETER ESTIMATES AND CASE STUdY RESUlTS

\begin{tabular}{|c|c|c|c|c|c|c|}
\hline \multicolumn{2}{|c|}{ Scenario } & & 67 & & & \\
\hline \multirow{2}{*}{\multicolumn{2}{|c|}{$\begin{array}{r}\text { Scene } \\
\text { Circumstance }\end{array}$}} & Ocean & Ocean & Land & Land & \\
\hline & & $12^{\circ}$ IFR & Winds & $7^{\circ}$ IFR & $45^{\circ} \mathrm{PBR}$ & \\
\hline \multicolumn{2}{|c|}{ Incidence angle } & $33^{\circ}$ & $53^{\circ}$ & $40^{\circ}$ & $40^{\circ}$ & \\
\hline \multicolumn{2}{|c|}{ Frequency band } & L & $\mathbf{K}$ & L & L & \\
\hline & $T_{y}$ & 105 & 180 & 215 & 198 & $\mathrm{~K}$ \\
\hline & $T_{h}$ & 80 & 120 & 170 & 188 & K \\
\hline & $T_{U}$ & 10 & 0.5 & 10 & .45 & $\mathrm{~K}$ \\
\hline \multirow{5}{*}{$\begin{array}{l}\text { Case } 1 \\
\text { (CC,HH) }\end{array}$} & $\overline{\hat{T}_{U}}$ & 8.63 & -0.68 & 8.25 & -41.1 & $\overline{\mathrm{K}}$ \\
\hline & error & -1.37 & -1.18 & -1.75 & 3.87 & $\mathrm{~K}$ \\
\hline & rel. error & $-14 \%$ & $-236 \%$ & $-18 \%$ & $-8.6 \%$ & \\
\hline & gain $(m)$ & 0.91 & 0.91 & 0.91 & 0.91 & \\
\hline & offset $(b)$ & -0.47 & -1.13 & -0.85 & -0.19 & $\mathrm{~K}$ \\
\hline \multirow{5}{*}{$\begin{array}{l}\text { Case } 2 \\
\text { (CC, HH, } \\
\mathrm{CH})\end{array}$} & $\hat{T}_{U}$ & 9.34 & 0.47 & 9.34 & -42.0 & $\mathrm{~K}$ \\
\hline & error & -0.66 & -0.03 & -0.66 & 2.96 & K \\
\hline & rel. error & $-6.6 \%$ & $-6.6 \%$ & $-6.6 \%$ & $-6.6 \%$ & \\
\hline & gain $(m)$ & 0.93 & 0.93 & 0.93 & 0.93 & \\
\hline & offset $(b)$ & 0 & 0 & 0 & 0 & $\mathrm{~K}$ \\
\hline \multirow{5}{*}{$\begin{array}{l}\text { Case } 3 \\
\text { (CC,HH, } \\
\mathrm{CN})\end{array}$} & $\bar{T}_{U}$ & 9.48 & -0.75 & 9.07 & -45.2 & $\mathrm{~K}$ \\
\hline & eтror & -0.52 & -1.25 & -0.93 & -0.21 & $\mathbf{K}$ \\
\hline & rel. епror & $-5.2 \%$ & $-249 \%$ & $-9 \%$ & $-0.5 \%$ & \\
\hline & gain $(m)$ & 1 & 1 & 1 & 1 & \\
\hline & offset $(b)$ & -0.52 & -1.25 & -0.93 & -0.21 & \\
\hline
\end{tabular}

IFR=ionospheric Faraday rotation

$\mathrm{PBR}=$ polarization basis rotation

for $\hat{T}_{U}$ and associated absolute and relative errors are listed. While under our assumptions expressions for $\hat{T}_{U}(25),(32)$, (38) and (42) can be summarized by

$$
\hat{T}_{U}=m T_{U}+b
$$

where $m$ is the $T_{U}$ gain and $b$ the $T_{U}$ offset. Values for $m$ and $b$ are given in Table II as well. These values are useful for determining the effects of the different calibration algorithms upon the errors.

For OSS, the errors are all $>0.5 \mathrm{~K}$, which is higher than desired for the Faraday rotation correction application. An error budget of $\sim 0.1 \mathrm{~K}$ is stated in [3]. Thus, the case- 4 calibration algorithm is most likely needed. Clearly crosspolarization errors are detrimental for OSW. In OSW the polarization contrast $T_{Q}$ is relatively large $(\sim 60 \mathrm{~K})$ and couples into $T_{U}$ via imperfect hybrid coupler amplitude balance. Because $T_{U}$ signatures of OSW are quite small $(<1 \mathrm{~K})$, any bit of $T_{Q}$ coupling into $T_{U}$ is detrimental. The error is slightly worse for Case 3 because the $T_{Q}$ leakage is emphasized by the polarimetric efficiency correction that occurs when the correlated noise source is included in the calibration.

The errors for SM(a) are similar to the case for OSS. The required brightness temperature uncertainty for SM, however, is at least an order-of-magnitude less stringent than for OSS, which will relax the $T_{U}$ calibration requirement. On the other hand, the absolute errors for SM(b) in Cases 1 and 2 are much larger than the other scenarios. The larger value of $T_{U}$ coupled with a $T_{U}$ gain of $\sim 0.9$ causes $\sim 3-\mathrm{K}$ error in $\hat{T}_{U}$. Case 3 , however, readily demonstrates the utility of the correlated noise source; the gain is corrected reducing the absolute error to a residual $0.2 \mathrm{~K}$, which is from cross-polarization coupling. 
TABLE III

UNCERTAINTY ANALYSIS RESULTS - CASE 4, OSS SCENARIO

\begin{tabular}{l|c||c|c||c}
\hline \multicolumn{2}{c||}{ Sensitivies $(\mathrm{K} / \mathrm{K})$} & \multicolumn{2}{|c||}{ Uncertainties (K) } & Products (K) \\
\hline$\partial T_{U} / \partial T_{H}$ & -0.0216 & $u_{T_{H}}$ & 0.5 & 0.0108 \\
$\partial T_{U} / \partial T_{C}$ & 0.0315 & $u_{T_{C}}$ & 0.5 & 0.0157 \\
$\partial T_{U} / \partial T_{C N}$ & 0.2010 & $u_{T_{C N}}$ & 0.5 & 0.1005 \\
$\partial T_{U} / \partial \hat{T}_{v}$ & 0.0169 & $u_{\hat{T}_{v}}$ & 0.5 & 0.0085 \\
$\partial T_{U} / \partial \hat{T}_{h}$ & -0.0268 & $u_{\hat{T}_{h}}$ & 0.5 & 0.0134 \\
\hline
\end{tabular}

A correlated noise source is probably needed when an antenna with a rotating polarization basis is used in the radiometer. Whether or not correction of $T_{Q}$ coupling using independent hot and cold looks is needed depends upon the expected amounts of ionospheric Faraday rotation and the overall error budget. Further investigation is needed before a conclusion can be made as to the optimal calibration algorithm (optimal in the sense of the least complex that meets requirements) for SM.

Because Case 4 has no systematic errors when the calibration noise temperatures are perfectly known, the simple numerical examples from above are not instructive. To generate errors using Case 4, we must assume that there is uncertainty in the calibration noise temperatures. This is typically done using the standard combined uncertainty expression:

$$
\begin{gathered}
u_{\hat{T}_{U}}=\left(\left(\frac{\partial \hat{T}_{U}}{\partial T_{H}}\right)^{2} u_{T_{H}}^{2}+\left(\frac{\partial \hat{T}_{U}}{\partial T_{C}}\right)^{2} u_{T_{C}}^{2}\right. \\
+\left(\frac{\partial \hat{T}_{U}}{\partial T_{C N}}\right)^{2} u_{T_{C N}}^{2} \\
\left.+\left(\frac{\partial \hat{T}_{U}}{\partial \hat{T}_{v}}\right)^{2} u_{\hat{T}_{v}}^{2}+\left(\frac{\partial \hat{T}_{U}}{\partial \hat{T}_{h}}\right)^{2} u_{\hat{T}_{h}}^{2}\right)^{\frac{1}{2}}
\end{gathered}
$$

where $u_{i}\left(i=T_{H}, T_{C}\right.$, etc.) are the standard uncertainties of the noise and brightness temperatures. Using the numerical values for the scene from the OSS scenario, the above terms are evaluated and presented in Table III. We use the same $0.5-\mathrm{K}$ value for all the calibration temperature uncertainties, which could arguably be too low or too high for any specific uncertainty. Nonetheless, using the same value is important so that the different contributions are not unduly weighted for the purpose of the example.

Of significant note in Table $\mathrm{II}$ is that the total uncertainty is dominated by the sensitivity to the correlated noise temperature. Its contribution is approximately ten times greater than those of the other sources. The uncertainty could thus be approximated:

$$
u_{\hat{T}_{U}}^{2} \approx\left(\frac{\partial \hat{T}_{U}}{\partial T_{C N}}\right)^{2} u_{T_{C N}}^{2}
$$

where by examining (42) the sensitivity is approximately

$$
\frac{\partial T_{U}}{\partial T_{C N}} \approx \frac{T_{U}}{T_{C N}^{\prime}}
$$

Then sensitivity is driven by the ratio of the value of $T_{U}$ to the value of correlated noise temperature. Having a larger value of correlated noise temperature would improve the calibration because the errors are interpolation errors when $\left|T_{U}\right|$ lies between 0 and $T_{C N}$. Of course, this only holds if increasing the value of $T_{C N}$ does not increase its uncertainty by a defeating amount. The principle is similar to two-look calibration of a conventional total-power radiometer, whereby an optimal hot load temperature can be found by trading the uncertainty and sensitivity [13].

\section{CONCLUSIONS}

We have proposed four calibration algorithms for microwave polarimeters that use hybrid coupler-based correlators. Because amplitude and phase imbalance in the radiometer components cause systematic errors in the third Stokes parameter measurement, careful consideration of calibration algorithms is necessary for reducing these errors. The four algorithms considered were (1) conventional two-look of hot and cold sources, (2) three looks of hot and cold source combinations, (3) two-look with correlated source, and (4) four-look combining methods 2 and 3 . Each of the four methods produce differing amounts of systematic error. When calibrating with method 1, the polarimeter has both gain and offset errors. Method 2 removes the offset error, while method 3 the gain error. Finally, method 4 compensates for all errors. The results obtained for the calibration methods where then applied to a case study radiometer in four different remote sensing scenarios. Applications for ocean surface salinity, ocean surface winds, and soil moisture with and without antenna feed polarization basis rotation were considered. This numerical study illustrated that the required calibration technique depends upon the application. Offset errors were more detrimental than gain errors for ocean surface winds remote sensing. Ocean surface salinity requires full calibration and soil moisture requires at least the correlated calibration source when used with a polarization basis rotation antenna system. Because the fourlook calibration method compensates for all systematic errors, a standard uncertainty analysis was performed to elucidate the dependencies on calibration source uncertainties. The largest sensitivity is to the correlated noise temperature. In particular, the value of the noise temperature and its uncertainty can be traded to achieve a minimum total uncertainty.

\section{REFERENCES}

[1] J. R. Piepmeier and A. J. Gasiewski, "High-resolution passive polarimetric microwave mapping of ocean surface wind vector fields," IEEE Truns. Geosci. Remote Sensing, vol. 39, no. 3, pp. 606-622, 2001.

[2] S. Yueh, W. Wilson, F. Li, S. Nghiem, and W. Ricketts, "Polarimetric measurements of sea surface brightness temperatures using an aircraft K-band radiometer," IEEE Trans. Geosci. Remote Sensing, vol. 33, no. 1, pp. 85-92, 1995.

[3] S. H. Yueh, "Estimates of Faraday rotation with passive microwave polarimetry for microwave remote sensing of Earth surfaces," IEEE Trans. Geosci. Remote Sensing, vol. 38, no. 5, pp. 2434-24388, 2000.

[4] A. J. Gasiewski and D. B. Kunkee, "Calibration and applications of polarization-correlating radiometers," IEEE Trans. Microwave Theory Tech, vol. 41, no. 5, pp. 767-773, 1993.

[5] N. Skou, B. Laursen, and S. Sobjacrg, "Polarimetric radiometer configurations: potential accuracy and sensitivity," IEEE Truns. Geosci. Remote Sensing, vol, 37, no. 5, pp. 2165-2171, 1999. 
[6] J. R. Piepmeier and A. J. Gasiewski, "Digital correiation polarimetry: Analysis and demonstration," IEEE Truns. Geosci. Remote Sensing, vol. 39, no. 11, pp. 2392-2410, 2001.

[7] Jaap Tinbergen, Astronomical Polarimetr;, Cambridge University Press, New York, 1996.

[8] International Standards Organization (ISO), Guide to the Expression of Uncertainty in Meusurement, Switzerland, 1995.

[9] A. Richard Thompson, James M. Morgan, and George W. Swenson, Jr., Interferometry and Symthesis in Radio Astronomy, John Wiley \& Sons, Inc., New York, $2^{\text {nd }}$ edition, 2001.

[10] F. T. Ulaby, R. K. Moore, and A. K. Fung, Microwave Remote Sensing. Active und Passive, Addison-Wesley Pub. Co., Reading, Mass., 1986.

[11] D.M. Le Vine and S. Abraham, "The effect of the ionosphere on remote sensing of sea surface salinity from space: absorption and emission at $L$ band," IEEE Trans. Geosci. Remote Sensing, vol. 40, no. 4, pp. 771-782, 2002.

[12] Barry N. Taylor and Chris E. Kuyatt, "Guidelines for evaluating and expressing the uncertainty of NIST measurement results," Tech. Rep. 1297, National Institute of Standards and Technology, Gaithersburg, MD, September 1994, Available on the World Wide Web at hitp://physics.nist.gov/Pubs/guidelines/contents. html.

[13] $P$. Racetle and $R$. Lang, "Comparative analysis of radiometer systems using non-stationary processes," in Proc. Int. Geosci. and Remote Sens. Symp. (IGARSS), Toronto, Canada, 2002, pp. 565-567.

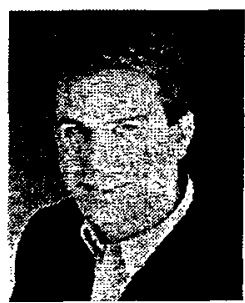

Jeffrey R. Piepmeier ( ${ }^{\prime} 89-M^{\prime} 99$ ) received the Ph.D. degree in electrical engineering from Georgia Institute of Technology in 1999. Previously, he received the M.S. degree in electrical engineeting from Georgia Tech in 1994 and the B.S. degree in engineering from LeTourneau University, Longview, Texas in 1993. From 1993 to 1994 he was a Schableford Fellow with the Georgia Tech Rescarch Institute. Piepneier was third place wimner in the 1998 IGARSS student prize paper competition. In 1999, he joined the Microwave Instrument Technology Branch at NASAs Goddard Space Flight Center, Greenbelt, MD. Dr. Piepmeier was recipient of an Excellence in Federal Career Gold Award (Rookie-ofthe-year) in 2000 and is a 2002 NASA Earth Science New Investigator. In 2000 he served as conference chair of the First Microwave Radiometer Calibration Workshop (MicroCa12000). He has been co-chairman of the Instrumentation and Future Technologies technical committee of the IEEE Geoscience and Remote Sensing Society since 2000 . He is a member of IEEE, URSI (Commission F), and the American Geophysical Union. His technical interests include passive remote sensing and technology development for microwave remote sensing. 